\title{
Surgical training in the midst of a pandemic: a distributed general surgery residency program's response to COVID-19
}

\author{
Graeme C. Hintz, MD, MEd \\ Katrina C. Duncan, MD, MPH \\ Emily M. Mackay, MD, MSc \\ Tracy M. Scott, MD, MHPE \\ Ahmer A. Karimuddin, MD, \\ MAEd
}

Accepted June 8, 2020

\author{
Correspondence to: \\ G. Hintz \\ Gordon \& Leslie Diamond Health Care \\ Centre \\ 11th floor 2775 Laurel St \\ Room 11125 \\ Vancouver BC V5Z 1M9 \\ graemehintz@alumni.ubc.ca
}

DOI: $10.1503 /$ cjs. 008420

\section{SUMmaRY}

The coronavirus disease 2019 (COVID-19) pandemic has had a substantial impact on surgical training. We describe some of the challenges brought on by the pandemic and our program's province-wide response to them. We focus specifically on residents' provision of service, education and wellness.

$\mathbf{T}$ he coronavirus disease 2019 (COVID-19) pandemic presents a unique health care challenge. As of July 14, 2020, there were more than 12.9 million cases and 570000 deaths worldwide. ${ }^{1}$ While much attention has been focused on pandemic response, surgical leaders have had to also consider the pandemic's impact on postgraduate surgical education. Resident physicians are uniquely positioned as both medical doctors and trainees, pursuing specialty training while providing patient care. At the University of British Columbia (UBC), the general surgery residency program has 44 residents distributed across more than 30 hospitals province-wide, including 2 quaternary centres in Vancouver. In the current pandemic, the balance between the service these residents provide and the protection of their educational environment has become more precarious. We discuss the challenges that the COVID-19 pandemic presents to resident training in terms of service, education and wellness and describe our response to those challenges (Fig. 1). ${ }^{2}$

\section{Provision of SERVICE}

The COVID-19 pandemic requires a sustained increase in personnel utilization without a clear end point. As such, we recalled all our distributed residents to Vancouver, where the demand was highest, in order to have a large reserve available. Residents were also taken off of rotations where most procedures had been cancelled or where clinical fellows were able to provide coverage. We determined the minimum staffing requirements of services where resident support was necessary and reassessed this continuously.

The communicability of severe acute respiratory syndrome coronavirus 2 (SARS-CoV-2), the virus that causes COVID-19, means that entire surgical teams could quickly be taken out of service and into self-quarantine. ${ }^{3}$ Therefore, we reformatted the acute care surgery (ACS) teams at our 2 quaternary centres to limit resident exposure to the virus, while still maintaining sustainable staffing. At Vancouver General Hospital (VGH), the ACS service was previously made up of 3 teams of 2 residents, each reporting to 1 surgeon. Residents were permitted to be on call only once every 4 days, leading to residents and staff from different teams frequently being put on call together. To limit this, we condensed into a 2 -team system with 2 junior-senior resident pairs reporting to 1 staff surgeon (Fig. 2). They worked for 2 weeks in isolation from others, and then had 1 week off, during which time they were replaced by another junior-senior pair. A similar system was developed at our 


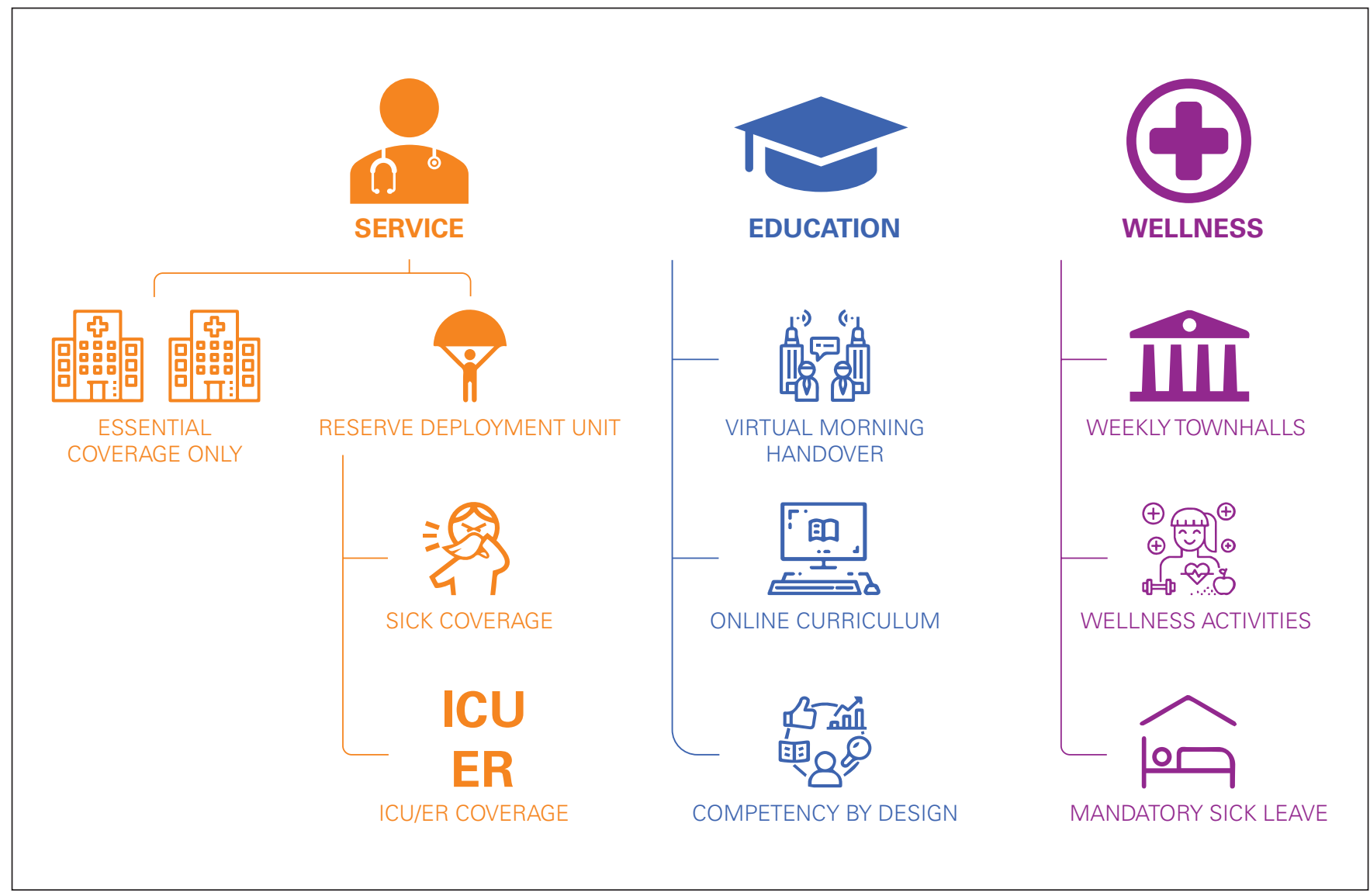

Fig. 1. General surgery residency program restructuring. The 3 main pillars of our program's restructuring in response to the coronavirus disease 2019 (COVID-190 pandemic were residents' provision of service, education and wellness. This figure illustrates the main components of each of these key elements. ER = emergency room; ICU = intensive care unit.

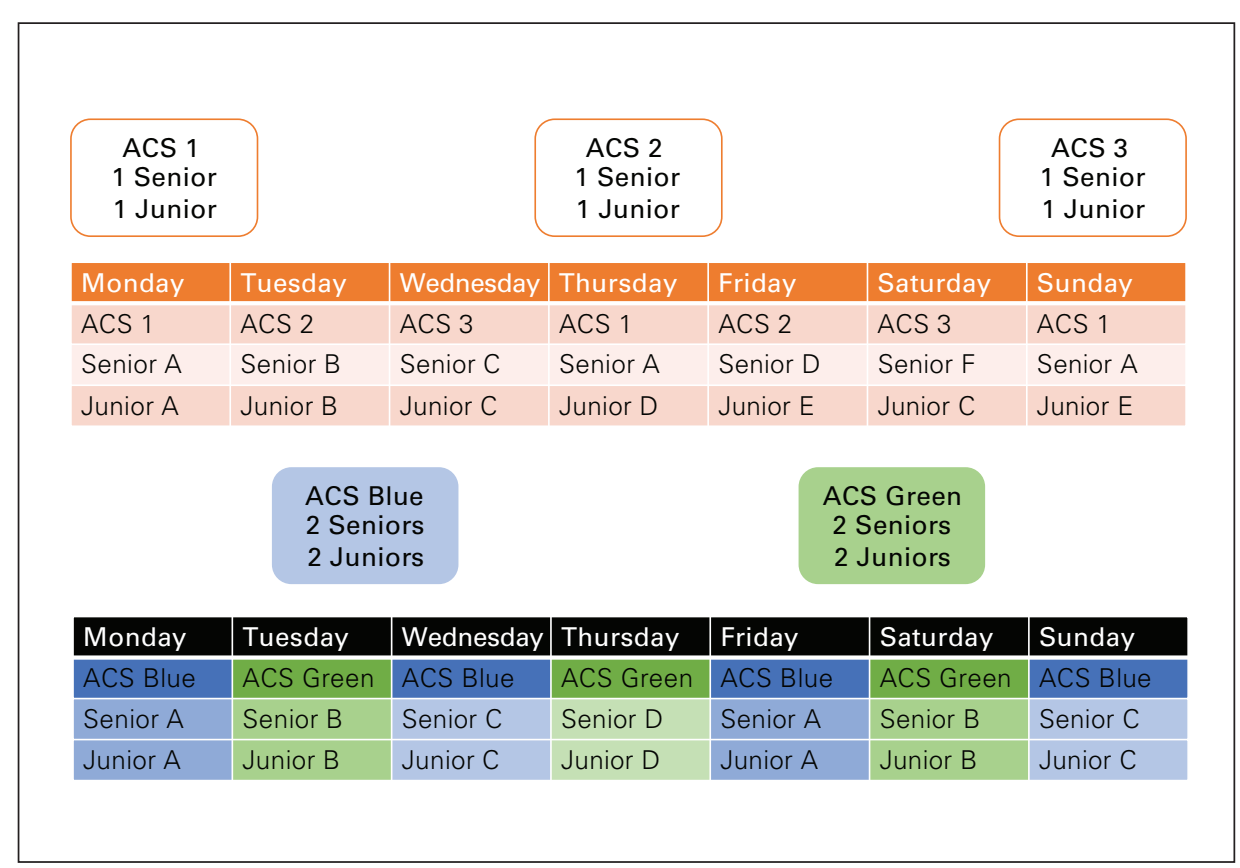

Fig. 2. Acute care surgery (ACS) restructuring. The top half of the figure shows the original 3-team ACS model, with its intemingling of team members for overnight call. The bottom half shows the restructured 2-team ACS model, with residents remaining in strict pairs for overnight call, helping to limit potential viral spread. second quaternary centre, ultimately limiting the number of residents in hospital while creating a pandemic-enduring system.

The remaining $40 \%$ of residents were divided between the intensive care unit (ICU) and the "reserve deployment unit," selfisolating at home to help when needed. We organized an online refresher course on intensive care for patients with and without COVID-19, and other critical care resources were also provided to residents. ${ }^{4}$

\section{Education}

The current pandemic has also affected resident education. Many residents had scheduled rotations shortened or altered, given elective surgery restrictions. Formal group educational 
activities, including rounds and educational sessions, have been cancelled. We have taken steps to limit this educational interruption. Our morning handover and teaching rounds now take place virtually. In fact, we have begun inviting general surgeons across the province to join these meetings to receive daily updates on the pandemic and review relevant literature. This collaborative space in which residents and attending surgeons can learn together will likely carry on after the pandemic. Our formal resident academic curriculum was already largely online in format, and it has not been difficult to move our junior resident curriculum to the virtual domain.

There are several unresolved educational issues. Residents in their final year have had their licensing exams postponed. Work is being done to ensure these residents are offered provisional licenses. Another challenge is how to make up missed rotations without extending the duration of training. This will require considerable focus on individualized learning plans to ensure key competencies are attained and may require earlier implementation of a competency framework to help assess educational needs.

\section{WeLLNeSS}

Intertwined with the delivery of excellent care and the ability to take advantage of educational opportunities is resident wellness. A lack of wellness has been associated with negative effects on both residents' and surgeons' personal and professional lives. ${ }^{5}$ In a pandemic, resident wellness is at further risk, given the increased work demands, the uncertainty of redeployment and the constant threat of personal or familial illness. To address these concerns, several steps were taken. Calling all residents back to Vancouver ensured that they were able to be with their families and support networks. Intraprovincial transportation has become difficult, with reduced ferry and airline services, and our early recall of residents guaranteed their return. Mandated return home also ensured residents could share the service-related burden.

Further, we have emphasized the importance of staying home if residents are feeling physically or mentally unwell. Residents and faculty were clearly informed that coming to work if unwell would be unprofessional. Some residents struggled with this, as they were conditioned to continue working during illness. Staying home when sick required an explication of the hidden curriculum underlying that behav- iour. We organized several activities to promote wellness. Weekly virtual town halls were initiated to share challenges, ensure familiarity with patient care protocols, and create a space in which to remain connected as a community. Guidelines were developed covering the expectations for patient care delivery, PPE use, and operative protocols. We ensured residents without an in-date N95 fit-test were expediently fitted. We stocked our resident lounge with nutritious snacks and meals for residents on call.

\section{Conclusion}

Postgraduate surgical training has been substantially affected by the COVID-19 pandemic, specifically within the realms of service, education and wellness. We rapidly restructured our program to meet these challenges. Further adaptations of our approach may be required as the pandemic continues. No residents have tested positive for the virus so far and we feel confident that, with these measures in place, we not only ensure continued patient care, but also continue to meet our trainees' educational and wellness needs.

Affiliations: From the Department of Surgery, University of British Columbia, Vancouver, BC (Hintz, Duncan, Mackay); and the Division of Surgery, St. Paul's Hospital, Vancouver, BC (Scott, Karimuddin).

Competing interests: None declared.

Contributors: All authors contributed substantially to the conception, writing and revision of this article and approved the final version for publication.

\section{References}

1. World Health Organization. Coronavirus disease 2019 (COVID-19) situation report-84. Geneva: World Health Organization; 2020 July 14. Available: https://www.who.int/emergencies/diseases/novel -coronavirus-2019/situation-reports/ (accessed 2020 July 15).

2. Quiros RM, Black E. Wellness in residency: a paradigm shift. In Stawicki SP, Firstenberg MS, et al. editors. Contemporary Topics in Graduate Medical Education. London: IntechOpen; 2018:1-11.

3. Li Q, Guan X, Wu P, et al. Early transmission dynamics in Wuhan, China, of novel coronavirus-infected pneumonia. $N$ Engl 7 Med 2020;382:1199-207.

4. Society of Critical Care Medicine. COVID-19 resources for nonICU clinicians. Mount Prospect (IL): Society of Critical Care Medicine. Available: https://covid19.sccm.org/nonicu.htm (accessed 2020 April 12).

5. Jackson T, Morgan J, Jackson D, et al. Trends in surgeon wellness (take a sad song and make it better): a comparison of surgical residents, fellows, and attendings. Am Surg 2019;85:579-86. 